\title{
A Range-free Localization Algorithm for WSN based on Error Correction and Multi-Hop
}

\author{
Xiu- wu Yu \\ Associate Professor \\ Environment and Safety \\ Department \\ University of South \\ China,China
}

\author{
Feng Zhang \\ PG Student Environment and \\ Safety Department University \\ of South China,China
}

\author{
Fei- sheng Fan \\ PG Student \\ Environment and Safety \\ Department University of \\ South China,China
}

\begin{abstract}
Considering analysis of localization algorithm in wireless sensor networks , and problem of lager error in Distance Vector-Hop(DV-hop)localization algorithm is mainly due to the estimation error of the distance between unknown nodes and anchor nodes, a localization algorithm based on error correction and multi-hop is proposed to reduce location error. The distance-per-hop of anchor is fixed by anchor nodes estimated and actual coordinates, unknown nodes receive limited number of received anchor nodes. According to the distance and the number of hops in a variety of circumstances to determine the distance of each hop distance, and then use the ratio of the square of distance to calculate the unknown node coordinates. Simulation results show that compared with DV-hop and WSGDV-Hop,EM-Hop algorithm can effectively improve the node localization accuracy and reduce the positioning error.
\end{abstract}

\section{Keywords}

WSN,Range-free localization,Error correction,Distance-perhop,Localization accuracy

\section{INTRODUCTION}

Wireless sensor network(WSN) can use to perceive, collect and process the information of related objects in the deployment area[1].Location is crucial to gather information in WSN, if wireless sensor network can not provide nodelocation, the information collected will be meaningless. Therefore,localization of information collection has become a key technique in wireless sensor networks study.

WSN positioning algorithm is divided into two types: rangbased and range-free[2]. Range-based algorithms need additional hardware support, mainly include Time of Arrival(TOA) [3] , Time Difference of Arrival (TDOA) [4] , Angle of Arrival(AOA ) [5], and Received Signal Strength Indicator (RSSI ) [6]. Range-free algorithms have low precision location, low cost and robustness. The typical rangefree algorithms include approximate Point-In-triangulation test(APIT ) [7], Centroid localization [8] algorithm and this paper will discuss the Distance Vector-Hop[9](DV-Hop ), etc.DV-Hop algorithm is simple and has no additional requirements for the network nodes. It has advantages of low overhead and can handle the case where a normal node has less than three neighbor anchors. However, when estimating the unknown node position, DV-Hop algorithm only uses average hop distance from the nearest anchor of unknown node, which is affected by the network topology and node density. DV-Hop has such disadvantages as low location accuracy and bad energy efficiency.Therefore, many algorithm based on DV-Hop have been proposed these past years[10 13] . In [10], the average every hop distance of anchor nodes is weighted by the error and the distance, the hop distance calculation method between unknown nodes to anchor nodes is selected by position judgment, and the calculated unknown nodes coordinates are optimized by improved genetic algorithm. The work in [11] presents an improved DV-HOP localization algorithm, where threshold M is introduced, it uses the weighted average hop distances of anchor nodes within $\mathrm{M}$ hops to calculate the average hop distance of unknown nodes.In [12] ,the author proposes Selective 3-Anchor DV-Hop, chooses the best 3 anchors based on connectivity parameters.It determine the average hop distance and estimates of hops with the nearest anchor. In [13] ,only one anchor node broadcasts its position coordinate in formation to other node,the ratio of position is introduced to reduce the position error after estimating coordinates of unknown nodes.

In order to solve defect that DV-hop usually selects average hop distance of the nearest anchor which brings great error , this paper presents a range free localization algorithm based on error correction and multi-hop( EM-Hop). Treating anchor nodes as unknown nodes, proposed algorithm estimates their coordinates,then fix them by actual coordinates to get the distance-per-hop, the unknown node receive information of limited anchor nodes. Depending on the relative distance and hop count to determine distance of each hop.Finally, the ratio of the square of distance is used to calculate the unknown node coordinates. The EM-Hop can provide higher location accuracy.

\section{EM-HOP LOCALIZATION ALGORITHM}

There are $\mathrm{N}$ nodes $A_{l}, A_{2}, \ldots, A_{N}$ distributed into two dimension region of $[0, /] \times[0, /], \mathrm{k}(\mathrm{k}<\mathrm{N})$ anchor nodes $A_{l}, A_{2}, \ldots, A_{k}$ are uniformly distributed in monitoring area,other nodes $A_{k+1}, A_{k+2}, \ldots, A_{N}$ are stationary unknown nodes.Assume that all nodes communication range is $R$.Nodes can only communicate with nodes in the range of its wireless range, and get information of its neighbors by forwarding message.

\subsection{Distance-per-hop}

First,each anchor node broadcasts beacon package $\left\{h_{i}, I_{i}\right\}$ including its location(coordinates)and the hop-count value.All nodes in communication range can receive information from anchor nodes. If the received data packets in the anchor node contain less hop-count, the hop-count in the table can be replaced with the hop-count of the received packet. If the packet is transmitted in the network, the hop-count will add 1 ,and transfer to its neighbor nodes.

Through this mechanism, all the unknown nodes in the 
network can obtain coordinates of anchor nodes and the smallest hop-count from anchor node. After that,unknown nodes will calculate the size of the hop-count of anchor nodes . $d_{i j}$ denotes Euclidean distance from node $i$ to node $j$. The average hop distance is shown as follows:

$$
\begin{gathered}
L_{i}=\frac{\sum_{j \neq i} d_{i j}}{\sum_{j \neq i} h_{i j}} \\
d_{i j}=\sqrt{\left(x_{i}-x_{j}\right)^{2}+\left(y_{i}-y_{j}\right)^{2}}
\end{gathered}
$$

where $\left(x_{i}, y_{i}\right)$ is coordinate of anchor node $i,\left(x_{j}, y_{j}\right)$ is coordinate of anchor node $i, h_{i j}$ denotes minimum hop-count between two anchor nodes.

Error produced by each hop of anchor node is related to the hop count and the distance between anchor node $i$ and anchor node $j$, which can be represented as follows:

$$
\Delta_{i}=\frac{\sum_{i \neq j}^{k}\left(d_{i j}-h_{i j} \cdot L_{i}\right) / h_{i j}}{(k-1)}
$$

Nodes are randomly distributed results that path from unknown nodes to anchor nodes is not uniform straight-line distance, besides error accumulation, the larger hop count between unknown nodes and anchor nodes is , the greater error is. so the average hop distance is modified ,the distance per hop as follows:

$$
H S_{i}=L_{i}+\Delta_{i}
$$

In DV-Hop ,only one average hop distance of nearest anchor to unknown nodes among all anchors, which can not represent surrounding situation around the unknown node network. Considering multiple anchor nodes of the average hop distance to reduce error, but too much data reception and transmission cause unnecessary energy consumption.In view of limited energy of nodes, normal nodes just need to record receive limited distance per hop of anchor nodes .

\subsection{Hop distance between unknown nodes and anchors}

Calculating the distance between an unknown node and an anchor node should take into account the hop count of them, as well as the average per hop error of anchor nodes.

In order to present this phenomenon, a typical example of network topology is introduced as shown in figure 1.This network distributes total 12 nodes randomly inside. The maximum communication range of all the nodes is set to $30 \mathrm{~m}$. Among the 11 nodes, 3 are anchors $A_{1} A_{2} A_{3}$ who already know their positions. The remaining units are normal nodes marked $U_{n}$. These normal nodes do not know their positions. The solid lines indicate that the two linked nodes are in each other communication range. And let's take $A_{1}$ as the nearest anchor of normal node $U_{n}$.

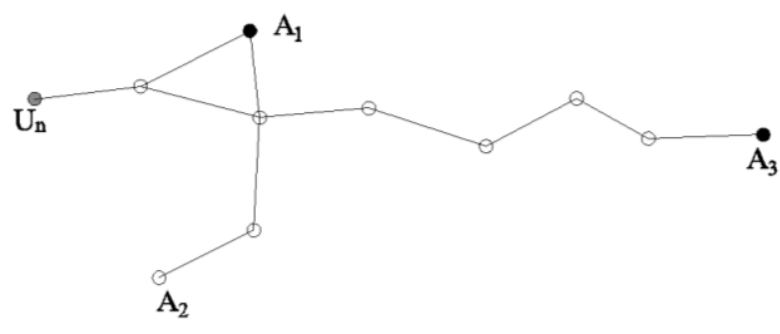

Fig 1:Example of nodes distribution

Estimated distances between anchors and unknown node $U_{n}$ denoted as $D_{i}$. Position relationship between unknown node and anchor node has the following three cases:

1. The nearest anchors are relatively close to the unknown node, which can well reflect the condition of its perimeter network,estimated distances between them as follow:

$$
D_{i}=H S_{i} \times h_{\min }
$$

where $h_{\min }$ is the minimum hop cont between nearest anchors and unknown nodes.

2. If hop count between unknown node $U_{n}$ and anchor nodes is greater than for minimum hop at least 5,the calculation of $D_{i}$ is shown as:

$$
D_{i}= \begin{cases}D_{(1, i)}+D_{1} & 0 \leq \angle A_{i} A_{1} U_{n}<\frac{\pi}{2} \\ D_{(1, i)}-D_{1} & \frac{\pi}{2} \leq \angle A_{i} A_{1} U_{n} \leq \pi\end{cases}
$$

where $\mathrm{D}(1, \mathrm{i})$ denotes the distance between the nearest anchor to unknown node and anchor node $i, \angle A_{i} A_{1} U_{n}$ is shown as follows:

$$
\angle A_{i} A_{1} U_{n}=\arccos \frac{H S_{i}^{2}\left(h_{\min }{ }^{2}-h^{2}\right)+D_{(1, i)}^{2}}{2 \cdot H S \cdot h_{\min } \cdot D_{(1, i)}}
$$

3. Since the third case is between the above two cases,path from anchor $\mathrm{A}_{2}$ to unknown node $U_{n}$ is fold line, dashed line is the shortest distance from unknown nodes to non-neighbor anchors .Hop count between them grater than 1,but there has low node density.If hop distance was adopted instead of straight-line distance, which leads to large error. Propagation range of each node is a circle of distance threshold centered in node $i$. 


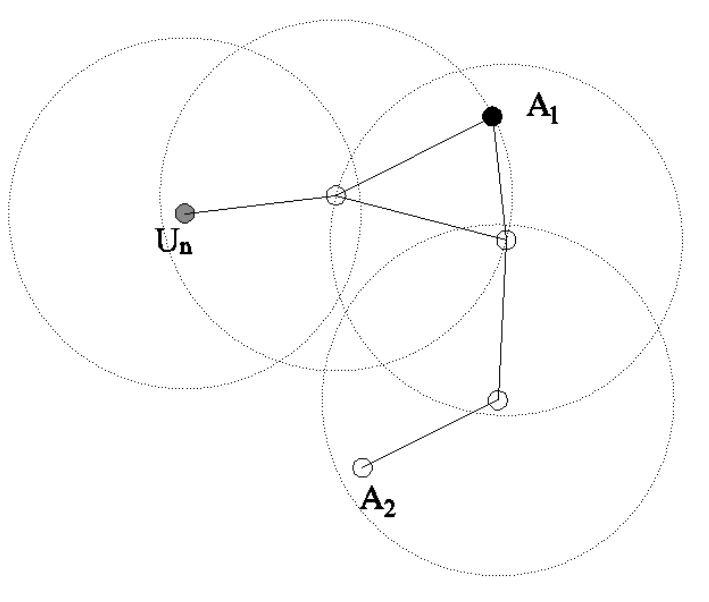

Fig 2:Shortest path of anchor A2 to other nodes

According to the "Hop-distance contradiction"of nodes ,if node $i$ and node $j$ are neighbor nodes which means their location distance is less than distance threshold.The minimum hop count from $U_{n}$ to anchor $\mathrm{A}_{2}$ is 4 ,then it can be obtained that: $D_{U A 2}<H S_{A 2} \times h<4 R$, as shown in figure 2 .

The calculation of $D$ is shown as:

$$
D_{i}=\omega \times H S_{i} \times h
$$

Scaling factor is used to reduce variation of estimated distance, which denoted as $\omega$, it can be defined as follows:

$$
\omega=\frac{h}{h+h_{\min }}
$$

\subsection{Estimated position of unknown nodes}

Estimated distances can be obtained which between normal nodes and limited anchors according to the preceding study.

Then, $U_{n}$ can calculate its estimated position by maximum likelihood algorithm based on $m$ estimated distance values $D_{l}, D_{2} \ldots D_{m}$. The true position of $U_{n}$ is denoted as $(x, y)$, and the positions of $\mathrm{A}_{1}, \mathrm{~A}_{2} \ldots . \mathrm{A}_{\mathrm{m}}$ respectively as $\left(x_{1}, y_{1}\right),\left(x_{2}, y_{2}\right) \ldots\left(x_{m}, y_{m}\right)$, ratio of the square of distance between unknown nodes and anchors is shown as follows:

$$
\mathrm{f}(x, y)=\sum_{i=1}^{m}\left(\frac{\left(x-x_{i}\right)^{2}+\left(y-y_{i}\right)^{2}}{D_{i}^{2}}-1\right)
$$

When $f(x, y)$ adopts extreme value, which make contributions to localization accuracy, the derivatives of $f(x, y)$ can be calculated with the following equation:

$$
\left\{\begin{array}{l}
\frac{\partial f}{\partial x}=2 \sum_{i=1}^{m} \frac{x-x_{i}}{D_{i}^{2}}=0 \\
\frac{\partial f}{\partial y}=2 \sum_{i=1}^{m} \frac{y-y_{i}}{D_{i}^{2}}=0
\end{array}\right.
$$

The coordinate of unknown node is estimated as follows:

$$
\left\{\begin{array}{r}
x=\frac{\frac{x_{1}}{D_{1}{ }^{2}}+\cdots \frac{x_{m}}{D_{m}{ }^{2}}}{\frac{1}{D_{1}{ }^{2}}+\cdots \frac{1}{D_{m}{ }^{2}}} \\
y=\frac{\frac{y_{1}}{D_{1}{ }^{2}}+\cdots \frac{y_{m}}{D_{m}{ }^{2}}}{\frac{1}{D_{1}{ }^{2}}+\cdots \frac{1}{D_{m}{ }^{2}}}
\end{array}\right.
$$

\section{SIMULATION AND ANALYSIS}

This section will verify the effectiveness of the proposed algorithm by simulating several scenarios and study the impact of different parameters on the proposed algorithm.Our simulations consisted of 2 parts:1)finding out the difference of relative positioning accuracy and average localization error between EM-Hop,DV-Hop and WSGDV-Hop.2)investigating how the key parameters,including number of anchor nodes and transmission range. The network consisted of 100 nodes deployed randomly and 5 40 anchor nodes in a region of $100 \times 100 \mathrm{~m}^{2}$.

\subsection{Evaluation metrics}

To analyze the simulation results, the following 2 metrics is used to evaluate the performance of EM-Hop.

1. Relative positioning accuracy is:

$$
\varepsilon=\frac{\sum_{i=1}^{\mathrm{n}} \sqrt{\left(x_{i}-x_{i}^{\prime}\right)^{2}+\left(y_{i}-y_{i}^{\prime}\right)^{2}}}{R \cdot N}
$$

where $\left(x_{i}{ }^{\prime}, y_{i}{ }^{\prime}\right)$ is estimation coordinate of unknown nodes , $\left(x_{i}, y_{i}\right)$ is real coordinate of unknown nodes.

2. Average localization error is:

$$
\hat{\varepsilon}=\frac{1}{N} \sum_{i=1}^{\mathrm{n}} \sqrt{\left(x_{i}-x_{i}^{\prime}\right)^{2}+\left(y_{i}-y_{i}^{\prime}\right)^{2}}
$$

where $\hat{\varepsilon}$ is the average localization error ; $\mathrm{N}$ denotes the number of all location-aware nodes.

\subsection{The impact of number of anchor nodes on positioning error}

The number of anchor nodes are taken 5, 15, 20, 25, 30, 35, 40 , the communication radius is $30 \mathrm{~m}$.This section compares the influence of average location error and relative positioning error for DV-Hop algorithm, EM-Hop algorithm and WSGDV-Hop in different proportion of anchor nodes.

As shown in figure 3 , as the number of anchor nodes increases, the average positioning error of 3 algorithms show a decreasing trend. Under the same conditions, the average location error of EM-Hop is smaller than DV-Hop and WSGDV-Hop. It can be seen from the figures below, when the number of anchor nodes rises from 5 to 15 , relative positioning error and average localization error of EM-Hop decrease greatly, when the anchor node is greater than 20 , the positioning error of the improved algorithm tends to stability.In average location error ,EM-Hop has been an 
average of $14.9 \%$ less than DV-Hop and $8 \%$ less than WSGDV-Hop.

\subsection{The impact of communication radius on positioning error}

The number of anchor nodes is 25 , the communication radius are taken 20, 25, 30, 35, 40.This section compares the influence of average location error and relative positioning error for DV-Hop algorithm,EM-Hop algorithm and WSGDV-Hop in different communication radius.

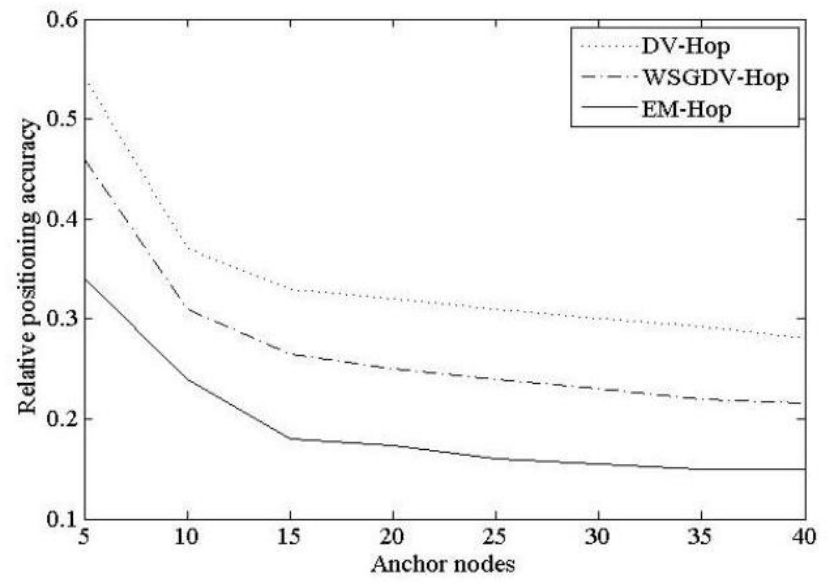

a)
As shown in figure 4, with the communication radius increases, the average location error and relative positioning error of 3 algorithms show a decreasing trend. Under the same conditions, the average location error and relative positioning error of EM-Hop is smaller than DV-Hop and WSGDV-Hop. In relative position error ,EM-Hop has been an average of 9.7\% less than DV-Hop and $4.7 \%$ less than WSGDV-Hop .

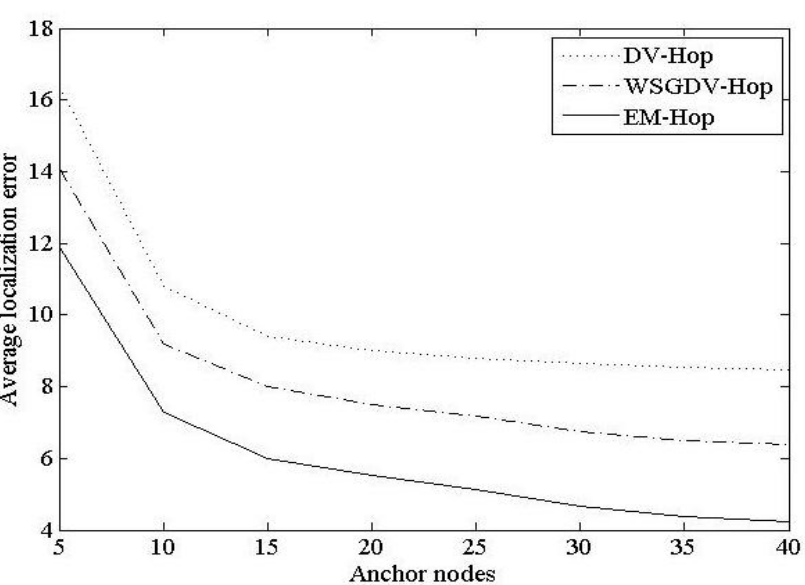

b)

Fig 3:Location error(range $30 \mathrm{~m}$ )

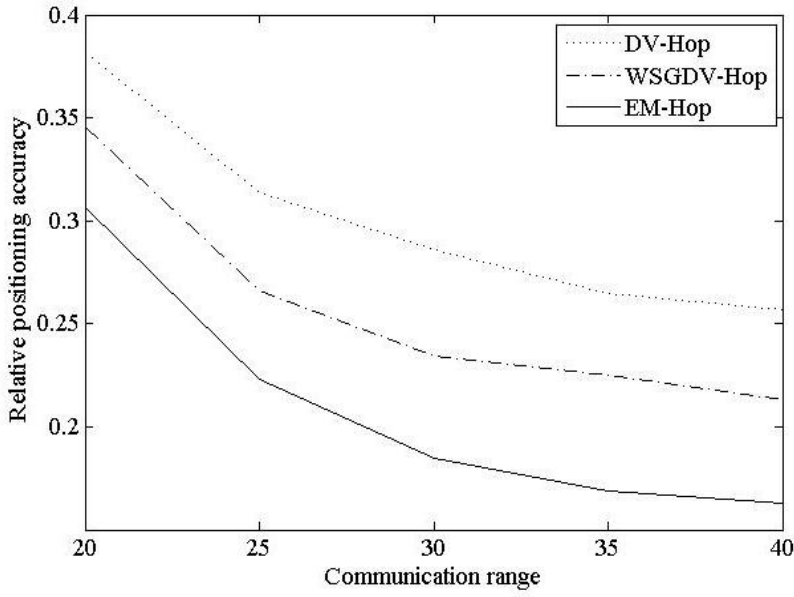

a)

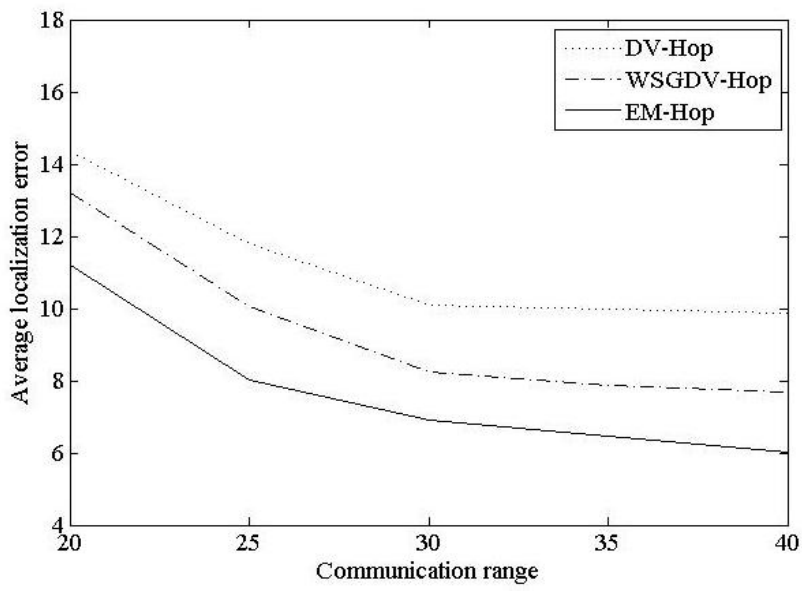

b)

Fig 4: Location error(number of anchor nodes is 25)

\section{CONCLUSION}

Localization is one of the substantial issues in wireless sensor network.This paper proposes a rang-free localization algorithm based on error correction and multi-hop. First, the unknown node collects threshold anchor nodes' distance perhop.Estimating these coordinates of anchor node as unknown nodes.According to the position relation between anchor nodes between normal nodes, the distance between them can be calculated.Then the ratio of the square of distance is introduced to calculate the coordinates of unknown nodes. Finally, this paper discusses the impact of the communication radius and the number of anchor nodes changing on the algorithm of the whole network positioning accuracy and average localization error.It can avoid overlapped error in a range-free localization model.Simulation results and analysis, comparing EM-Hop with DV-Hop and WSGDV-Hop,show our proposed algorithm has better results in the localization accuracy and perform better.

\section{ACKNOWLEDGMENTS}

This work was supported in part by the Key Research Projects of Hunan (2015SK2005), the Key Scientific Projects of Hunan Education Committee (15A161), the Natural Science Foundation of Jiangxi Provinces (20122BAB201050); Technology Supporting Project of Jiangxi Department of Science and Technology (20121BBG70065, Technological Projects of Jiangxi Education Committee (GJJ12667), the Doctoral Scientific Research Funds of University of South 
China (2013XQD12) and the Scientific Research for Graduate Students of Hunan CX2016B453.

\section{REFERENCES}

[1] Pinghui Zou,Yun Lin.A data-aggregation scheme for WSN based on optimal weight allocation[J].Journal of Networks,2014,9(1): 100-107.

[2] M. Golestanian, C. Poellabauer. Localization in heterogeneous wireless sensor networks using elliptical range estimation[C]// International Conference on Computing, NETWORKING and Communications. IEEE Computer Society, 2016:1-7.

[3] Harter A, Hopper A, Steggles P, et al. The Anatomy of a Context-Aware Application[J]. Wireless Networks, 2002, 8(2):187-197.

[4] Girod L, Estrin D. Robust range estimation using acoustic and multimodal sensing[C]// Ieee/rsj International Conference on Intelligent Robots and Systems, 2001. Proceedings. IEEE, 2001:1312-1320 vol.3.

[5] Niculescu D, Nath B. Ad Hoc Positioning System (APS) Using AoA $[\mathrm{J}]$. Proceedings - IEEE INFOCOM, 2003, $3: 1734-1743$

[6] Girod L, Bychkovskiy V, Elson J, et al. Locating Tiny Sensors in Time and Space: A Case Study[C]// IEEE International Conference on Computer Design: Vlsi in Computers and Processors. IEEE Computer Society, 2002:214-219.

[7] He T, Huang C, Blum B M, et al. Range-free localization schemes for large scale sensor networks[C]// International Conference on Mobile Computing and NETWORKING. ACM, 2003:81--95.

[8] Bahl P, Padmanabhan V N. RADAR: an in-building RFbased user location and tracking system[J]. Institute of Electrical \& Electronics Engineers Inc, 2000, 2:775--784.

[9] Niculescu D, Nath B. Ad hoc positioning system (APS)[C]// Global Telecommunications Conference, 2001. GLOBECOM '01. IEEE. 2001:2926--2931.

[10] Cheng Chao,Qian Zhi-hong,Fu Cai-xin,et al.Genetic Optimization DV-Hop Localization Algorithm Based on Error Distance Weighted and Hop Algorithm Selection.[J]. Journal of Electronics \& Information Technology , 2015(10):2418-2423.
[11] Hu Y, Li X. An improvement of DV-Hop localization algorithm for wireless sensor networks[J]. Telecommunication Systems, 2013, 53(1):13-18.

[12] Gui L, Val T, Wei A, et al. Improvement of range-free localization technology by a novel DV-hop protocol in wireless sensor networks[J]. Ad Hoc Networks, 2015, 24:55-73.

[13] Li Y. An Improved DV-Hop Localization Algorithm Based on Energy-saving Non-ranging Optimization[J]. Journal of Networks, 2014, 9(11).

[14] So-In C, Katekaew W. Hybrid Fuzzy Centroid with MDV-Hop BAT Localization Algorithms in Wireless Sensor Networks[J]. International Journal of Distributed Sensor Networks, 2015.

[15] Chao J, Han G, Zhu C, et al. Performance evaluation of DV-hop localization algorithm with mobility models for Mobile Wireless Sensor Networks[C]// Wireless Communications and Mobile Computing Conference. IEEE, 2013:813-831.

[16] Sivakumar. S , Venkatesan R, Karthiga. M K M. Error Minimization in Localization of Wireless Sensor Networks using Genetic Algorithm[J]. International Journal of Computer Applications, 2012, 43(12):16-20.

[17] Alavinia R, Zhu Z, Zhang S. Design and Simulation of a Meteorological Data Monitoring System Based on a Wireless Sensor[J]. International Journal of Online Engineering, 2016, 12(5).

[18] Ramazany M, Moussav Z. Localization of Nodes inWireless Sensor Networks by MDV-Hop Algorithm[J] Arpn Journal of Systems \& Software, 2012, 84(10):455464.

[19] Huang B, Yu C, Anderson B D O. Understanding Error Propagation in Multi-hop Sensor Network Localization[J]. IEEE Transactions on Industrial Electronics, 2012, 60(12):5811-5819.

[20] Song G, Tam D. Two novel DV-Hop localization algorithms for randomly deployed wireless sensor networks[J]. International Journal of Distributed Sensor Networks, 2015, 11. 